\title{
Sexually transmitted infections in teens and youth challenge science and technology
}

\begin{abstract}
Youth and adolescence have traditionally been defined as ages of transit between childhood and adulthood. During adolescence, the vulnerability to contracting sexually transmitted infections (STIs) increases due to sexual and social risk behaviors. Insufficient perception Risk, beliefs and false myths about the different objects and phenomena of reality and inadequate attitudes regarding these phenomena constitute reasons for the increasing dissemination and difficult control. The problem of STIs is a global concern and Cuba does not escape from this situation. For science and technology, the problem is related to the fact that STIs hinder fertility and natality by controlling the demographic dilemma in Cuba and increasing the biomedical and socioeconomic costs associated with them. The objective of the research is related to the answers and technological and scientific solutions that have been developed for the protection of sexual and reproductive health.
\end{abstract}

Keywords: adolescence, science, technology, population, concern, childhood
Volume 8 Issue 3 - 2019

\author{
Damarys Chacón O'farrill,' Alba Cortes \\ Alfaro $^{2}$ \\ 'Department of Gynecology, Polyclinic Luis Augusto Turcios \\ Lima, Cuba \\ ${ }^{2}$ Department of Epidemiology, National Institute of Hygiene, \\ Cuba \\ Correspondence: Alba Cortes Alfaro, Second Degree \\ Specialist in School Hygiene. Masters in Epidemiology, Assistant \\ professor and researcher, National Institute of Hygiene, \\ Epidemiology and Microbiology, Havana, Cuba, \\ Email albac@inhem.sld.cu
}

Received: April 16, 2019 | Published: May 07, 2019

\section{Introduction}

WHO considers adolescence as the period understood life between 10 and 19 and is assumed as a "psychological age" where it is necessary to devise psychological development "as a process that does not happen automatically nor determined fatally by maturation of the organism but is primarily a historical determination social. ${ }^{1,2}$ Teenagers are considered a vulnerable group because of sexual and social behaviors that increase susceptibility to a disease or a poor health, as well as being associated with the perception of erroneous risk of the possibility of contracting the infection or be immersed in pregnancy (perceived vulnerability). ${ }^{3-7}$ Sexually transmitted infections (STIs) include a number of diseases, infectious nature in which sexual transmission is epidemiologically relevant, although some of them is not the most important transmission mechanism. It is now known more than 30 STIs, some of them not curables. ${ }^{8}$

\section{Magnitude of the problem}

WHO estimated 333 million new cases sexually transmitted diseases curable in 15 to 49 years, most countries in developing including member countries Community European? Moreover recent estimates report that occur each year in the world more than 340 million cases of curable vaginal infections, whose manifestation syndrome vaginal susceptible flow of effective treatments and that at least one million infections occur each day. Only for Latin America $\mathrm{Y}$ the Caribbean were infected between 35 and 40 million cases with more than 100,000 infections per day average. In EE UU. 15 million people are infected with one or more ITS annulment. La highest proportion seen in South and Southeast Asia, followed by sub-Saharan Africa and Latin America and the Caribe. En relation to HIV in the world around 38.6 million people suffer; 10.3 million of them are young people aged 15 to 24 years, $42 \%$ were newly infected. Moreover, $50 \%$ of new infections, nearly 6,000 daily, occur in young people. If gender analysis is seen infecting an increasing number of women, and significantly earlier than in the case of varones ${ }^{9}$ ages. In Cuba about 50000 cases of STIs, with an upward trend in recent years are reported annually. Among the most common are gonorrhea, syphilis, NGU, condyloma acuminata, trichomoniasis, genital herpes simplex and HIV / AIDS. The HIV/AIDS has been characterized by a slow and sustained increase with increased incidence in the last five years; men who have sex with men are the group most vulnerable. The predominant form of infection is transmission sexual. ${ }^{10}$

Statistical Yearbook of Cuba in 2017 shows that the incidence of syphilis increased. In 2013 a total of 3302 to 4046 in 2014 and 2015: 4582, in 2016 a total of 5008 in 2017: 5088 male dominance always with. As for the incidence rate of gonorrhea in 2014: 4116 AND 2015: 3961 in 2016: 3547, of which 704 adolescents, 2017: 2904. Down ward trend is observed, the female is always the most affected. In the 2016 yearbook it is reported that Condy loma infection as a notifiable disease in adolescence 1274 figures reached a prevalence of 847 in féminas. ${ }^{11,12}$ The incidence of AIDS: oscillating trend as in 2000 reported 258 cases in 2013: 316, in 2014 amounted to 640 and from 2015 the trend is to decrease: 398 , in 2016: 319 and 2017: 168 the male was affected more cuantía. ${ }^{11,12}$

As for the sociodemographic characteristics of the population of adolescents in Cuba can be seen representing $11.92 \%$ of the total population with a $13 \%$ reduction between 2000 and 2010 given the demographic dynamics of the country descends birth and increases the aging population. ${ }^{13}$ discreetly dominated by male adolescents $(689,867,51.50 \%)$ over female $(48.49 \% 649,468$ on); $15.8 \%$ of women aged 15-19 had been married or in union and $5.9 \%$ before 15 years. $12.9 \%$ reported having had sex at this age. Although there is a contraceptive coverage in our country $74 \% 40.1 \%$ do not use contraception, For all the questions raised can pronounce that the problem of science and technology related to STIs is given by the demographic dilemma in Cuba and biomedical and socioeconomic costs associated with éstas. ${ }^{14}$

\section{Developing}

The population pyramid is inverted with fewer young people, if you have more chance of ITS sequelae that may occur will ruin the fertility, birth rate and increased morbidity and mortality in this population so the technological scientific problem is aimed at 
preventing STIs, encourage early diagnosis and early treatment in addition to promoting information with positive changes in attitudes, perception of irrigation and beliefs taking into account gender, sexual rights and sociocultural.

1. Fertility situation in $\mathrm{Cuba}^{13,15}$

2. Latin American country with therate natalidad more low: 11.2 (2015)

3. Latin American country with the TFR more baja-1.6 (2015)

4. Reduction in the number of births

a) Smaller population of women in the appropriate age for motherhood.

b) Reduced fertility in women between 20 and 29 years.

c) Shift in fertility towards the extremes of age, under 20 and over 35 years, but this is not enough to offset the decline in fertility between 20 to 29 years.

By 2020 there were $21.5 \%$ of older adults in 2030 reached $30 \%$ and by 2050 will account for $36.2 \%$, then: we will be the oldest population in the world with population declines country?

Objective: Demonstrate how Cuba has faced the challenge of increasing STIs in adolescents and young people through the development of science and technology taking as a basis the guidelines of the Politics, Economics and Social Party and Revolution and Sustainable Development Goals for 2030. ${ }^{16,17}$

The authors make a compendium of literature, making visible scientific technological work. Biomedical costs and Socioeconómicos14To cushion the costs have set working towards several lines.It is imperative to protect future fertility of Cuban women and especially since the most tempranas. Las ages suffer them sequelae after STI in women is more severe, due to the anatomical and physiological factors and the likelihood of delay in treatment.

\section{Among the consequences they are cited}

Infertility: It is one of the most common complications, results from damage to the fallopian uterine the ascent to the upper genital tract of the causative agents of gonorrhea, Chlamydia or possible bacterial vaginosis, which causing pelvic inflammatory disease (PID). Numerous data indicate that 10 to $40 \%$ of women with chlamydial or gonococcal infection untreated symptomatic PID evolve and more than $25 \%$ of these result infértiles. ${ }^{18}$ Cuba has deployed an enormous economic effort in acquiring material resources for the diagnosis and treatment of these, as well as developing human resources for the care of infertile couples in assisted reproduction consultations tech. The whole country is governed by the treatment protocol of infertile couples at different levels of care, organization and ensuring prompt and appropriate resolution according to the causes of this entity.

Ectopic pregnancy (tubal): Is STI complication, potentially fatal, is also often associated with a previous pelvic inflammatory disease (PID) and incomplete tubal occlusion light. Women who have had PID are between 6 and 10 times more likely to have an ectopic pregnancy than women who have never suffered from infection of the upper genital tract. EPI after a first pregnancy is ectopic pregnancy in about $6 \%$ of women. Currently ectopic pregnancy is one of the most important causes of maternal death in the 2017 statistical yearbook reported maternal deaths from this cause in 2 cases in 2015 and 2016 and 1 in $20178 .{ }^{12}$ Cuba has developed guidance for early diagnosis of ectopic pregnancy through protocol and material resources it has been earmarked as the pregnancy test that previously were imported and Heber Fastline is currently produced in Heber Biotech SA Havana.

Cervical cancer: Available evidence shows that the human papillomavirus (HPV) plays the leading role in causing cancer cervical, ${ }^{19,20}$ as part of an early diagnosis program for early detection of cervical cancer is created with the production of diagnostic kit. Cuba produces Colposcopia equipment that facilitates the diagnosis and timely treatment of pre malignant lesions.

Adverse pregnancy outcomes: In addition ectopic pregnancy, unfavorable outcomes related to STIs including fetal loss (abortions or stillbirths), low birth weight (premature delivery or intrauterine growth retardation) and connatal infection (blindness potential, pneumonia and mental retardation) are indicated.

Cuba several doctors to work among those who are drawn protocols Abortos: ${ }^{21}$

1) Methodological guidelines for the safe conduct of voluntary termination of pregnancy or spontaneous Abortions admas updating action protocols are created guides.

a) Ultrasonography with early diagnosis at 12 weeks.

b) VACUM (vacuum machines), Syringes Karman cannulated (5 millimeters, imported technologies)

c) Techniques abortion with Misoprostol (TVE)

d) epidemiological requirements with sterilization procedures and high level disinfection in the reusable material

e) Skilled personnel (accredited previous training).

2) Low birth weight (premature delivery or intrauterine growth retardation)

a) Medical Biotechnology and Pharmaceutical Industry:

b) Sufarcendesarrollado by the National Center for Animal Health (CENSA) for the treatment of hyaline membrane.

c) Progesterone Deposit for lung maturation.

d) Cervical pessary.

e) Intensive care for monitoring and therapeutic technologies requiring adequate medical transport including enabled with advanced technology for the treatment of neonate.

f) Neonatologist scientific development that should acquire through training and continuous development of their skills.

\section{To prevent STIs}

Our health system aims to reduce the number of abortions in general and under 20 years in particular. Ensure effective contraception recommends that allows planning of offspring at will and according to social and family requirements. The municipal family planning consultations performs this function. It remains: The national strategy aims condoms increased marketing, supply, diversity, logistics and financing of these projects and market research and segmentation of the population (social marketing of condoms)23-42. Diagnostic STI: Advanced Technologies. Microbiology and the diagnosis of 
infectious diseases is one of the areas of biomedical science where DNA technology is having more impact. The application of the techniques of molecular biology to the clinic is revolutionizing they diagnose a large number of diseases characterized by infecciosas. Se faster (minutes or hours to days differences conventional diagnostic tests), are highly specific and simple, which facilitates automation. It stands out among ellas:22

a. PCR: for diagnosis of Cytomegalovirus Virus and viral hepatitis $\mathrm{B}$ and $\mathrm{C}$.

b. ARNr: for chlamydia and mycoplasma.

c. The ELISA for detecting antibodies against human immunodeficiency virus.

d. Genetic screening for the detection of genetic and chromosoma diseases highlighted in molecular medicine and biotechnology.

e. Studies fourth-generation antibiotics because the phenomenon of antimicrobial resistance in STI in addition to continuing the search for an HIV vaccine is observed.

f. The tests based ontechnologylatex particle agglutination linked to specific antibodies, with visual observation or the microscope; it has been used for several years and maintains their use today. It was developed after solid phase technology, comprising the test strips wide application.

To reduce the incidence of STIs in adolescents and youth is identified as an essential aspect of promoting salud. ${ }^{23} \mathrm{Health}$ promotion is to provide people with the means to improve health and exercise greater control over misma. ${ }^{24}$ Promotion section of the National School of Public Health (ENSAP) appreciates it as a new approach, qualitatively superior to traditional public health, as a new paradigm. For WHO/PAHO is either a strategy or a special function of public health. One aspect that emphasizes health promotion is self-defined as a practice of activities by individuals in favor of itself to sustain life, health and welfare, respecting the right of each person to maintain control Individual its own pattern of life. ${ }^{25}$ The authors consider that it is necessary to stimulate people from an early age the need to assume the construction of their health responsibly. It is proposed that the unification of the health system and education should occur as it is at school where patterns of healthy habits that help the individua to choose lifestyles and avoid risk factors affecting negatively the quality of life are acquired. Health promotion indisputably part of the educational process whose function is to form an integral way to alumnos. ${ }^{26}$ This was reflected in the Ministerial Resolution 1/97 and the 139/2011: Education Program sexuality with gender and sexual rights in the national education system with the importance of MINEDMINSAP interrelation with medical integration-teaching. ${ }^{27-30}$ It gives response to the Guidelines for the Political, Economic and Social Party and the Revolution adopted on November 1, 2010: Year 52 of the Revolution, in the framework of the 6th Congress of the Communist Party of Cuba and Sustainable Development Goals 2030. ${ }^{31}$ guidelines of the Politics, Economics and Social Party and Revolución16: Policy Science, technology, innovation and the environment. Article 123 It should sustain and develop the results achieved in the field of biotechnology, medical pharmaceutical production, the software industry, educational technologies, scientific services and value-added technology, bioinformatics and nanotechnology. ${ }^{32}$

\section{Social policy}

Article 129 Continue preserving the conquests of the revolution, such as access to health care, education, culture, sports, recreation, social security and welfare protection to people in need. Article 132 To continue the improvement of education, health, culture and sports, for which it is essential to reduce or eliminate excessive spending in the social sphere.

\section{Education}

Article 133 Further progress in raising the quality and rigor of the educational process and achieve further improvement of existing capacity, from the establishment of joint centers that favors the formation of the different levels of education in line with the needs. This involves resizing centers and achieves better utilization of the workforce. Article 134 Form in each territory which is required to meet the needs of schools at different levels of education teachers. Article 135 To promote the role of the teacher in front of the classroom and get equipment and audiovisual material that complements the educational work are used rationally.

\section{Health}

Artículo143. Enhance the quality of service provided, the satisfaction of the population and improving working conditions and health care personnel. Ensure efficient resource update, savings and eliminating unnecessary expenses. Article 144 Rearranging territorially services and efficiently employ technology that is available, enhance the clinical diagnosis and rational use of supplementary means of studies, especially the more expensive technology. Consolidate and require the use of protocols for diseases.

\section{Guideline for the main branches}

Article 203 Consolidate the pharmaceutical and biotechnology industry as one of the activities of greater export capacity of the economy and bring new products to the domestic market to replace imports. Sustainable Development Goals for 203017.

1) Objective 3. Ensure a healthy lifestyle and promote wellness for everyone at all ages.

2) Objective 4 . To ensure an inclusive, equitable and quality education and promote learning opportunities throughout life for everyone.

3) Objective 5. Achieve gender equality and empower all women and girls.

4) Objective 16. To promote peaceful and inclusive societies for sustainable development, facilitating access to justice for all and to create effective, accountable and inclusive institutions at all levels.

The authors consider to be maintained and improve family planning services and reproductive risk existing and develop new services in institutions responsive to the needs identified for adolescent care, continue especially at the level of primary care management and control of preconception reproductive risk, promote knowledge, encourage reflection and participation of the individual, the couple, the family and the community in matters relating to family planning, so that they adopt responsible behavior in this area, continue the educational work with the aim of increasing knowledge and guidance to adolescents and young people responding to their needs relation to the overall health. 


\section{Conclusion}

The increase of STIs in adolescents and young causes damage to sexual and reproductive health to be affected somehow fertility and birth. In Cuba acts directly on the population pyramid therefore it does not occur adequately the population replacement. Advances in science and technology to develop positively influence protocols appropriate and effective action, make effective diagnosis, precise treatments are given to them and avoid the consequences and complications. It promotes interdisciplinarity in health promotion. This is in response to the Guidelines Cuba party and launches responses to Agenda 2030.

\section{Acknowledgments}

None.

\section{Conflicts of interest}

The author declares there are no conflicts of interest.

\section{References}

1. Cruz Sánchez F, B Alonso Rodriguez, Alonso Uría RU. Childcare in adolescence. Havana: Ministry of Public Health; 2014. pp.15-16.

2. Cruz Sánchez F, et al. Adolescence. Pediatrics. Havana: Medical Sciences; 2011. 5 p.

3. Santillano Cardenas I. Integral Education of sexuality in childhood, adolescence and youth. Conceptual approach. Guerrero and looks Reflections on sexuality in childhood, adolescence and youth. Havana: CENESEX; 2016. 45 p.

4. MI Mesa Alvarez Dominguez Gomez J, Navarro Torre LM. Factors related to the spread of sexually transmitted infections in adolescence. Revhabanciencméd. 2014;13(2):131-130.

5. World Health Organization. Sexually transmitted infections. Fact Sheet No. 110. 2016. 8 p.

6. Guerrero Borrego N, Perez M. Enriquez Integral Education of sexuality in childhood, adolescence and youth. Conceptual approach. In reflections and perspectives on sexuality in childhood, adolescence and youth. Havana: CENESEX; 2016. pp.11-32.

7. MI Mesa Alvarez Dominguez Gomez J, Navarro Torre LM. Factors related to the spread of sexually transmitted infections in adolescence. Revhabanciencméd. 2018;1(2):187-198.

8. Cutie E. sexually transmitted infections. Obstetrics and gynecology. Havana: Medical Sciences; 2004. 285 p.

9. World Health Organization. HIV AIDS. 360 sheet $N^{\circ}$. Washington: WHO; 2013.

10. Health Statistics Yearbook 2012. Republic of Cuba. National Directorate of Medical Records and Health Statistics. MINSAP. La Havana; 2012. $158 \mathrm{p}$.

11. Ministry of Public Health. Health Statistics Yearbook 2015. Havana: Medical Sciences; 2018. 208 p.

12. Health Statistics Yearbook 2017. Republic of Cuba. National Directorate of Medical Records and Health Statistics. 2018.

13. Diaz Machado A, F Sanchez Cruz Leon Reyes S, Bess Constantèn S, et al. Adolescents in Cuba. Indicators in their health, 2016. Adoleca magazine. 2017;121(5):9-34.
14. Sexually transmitted infection. In: Head Cruz E, CuTiE Leon ES, editors Manual procedures in gynecology. Havana: Medical Sciences; 2006. 90 p.

15. Casanova Fabelo L, EstevezGarcia KR. Cuba in the educational agenda post 2015. Study Rev. 2016;1(20):28-40.

16. Draft guidelines, economic and social policy. The 6th Congress of the Communist Party of Cuba. 2010. pp.18-25.

17. Draft final document of the summit of the United Nations for approval of the development agenda after 2015. PAHO/WHO.

18. Study and treatment of the infertile couple. In: Head Cruz E, CuTi E Leon E, Alba Santisteban, editors. Manual procedures in gynecology. Havana: Medical Sciences; 2006. 57 p.

19. Andrade G. Cervical Cancer and Human Papillomavirus (HPV). 2011.

20. Diagnosis and behavior in cervical cancer. In: Head Cruz E, CuTi E Leon E, Alba Santisteban, et al. Manual procedures in gynecology. Havana: Medical Sciences; 2006; 162 p.

21. WHO definition of unsafe abortion. Boletín of the World Health Organization. 2014;92:155.

22. A Salgado J. Barquinero molecular medicine and biotechnology. Editorial: Pharmaceutical Chemistry Bayer. 2015. 16 p.

23. Brooks R A, Allen VC. Perceptions of HIV and intentions to adopt preexposure among black men prophylaxis Who Have sex with men in Los Angeles. 2015. AIDS Education and Prevention. 2016;28(5):365-377.

24. Site STI-HIV-AIDS. Archive for 'STIs and sexual behavior' Cuban teenagers. Panorama Health. 2013.

25. Benavides K, Alfaro DP. Educational mediation strategies for education about sexually transmitted infections in adolescents. Current Rev. Nursing in Costa Rica. 2016;31:48-59.

26. Lima Valley adla educational research. Another look. Central Institute of Educational Sciences. Cub: Havana city; 2010. 35 p.

27. Fernando Marqués. Theoretical framework for the promotion and health education. Sagepub.1999;5(2):242-255.

28. Castellón de la Plana. Differential effectiveness of different strategies for AIDS prevention promotion of safe sex and performing diagnostic HIV testing in university students [Master's thesis]. 2017. 94 p.

29. Program Sexuality Education Gender and Sexual Rights in the national education system. Ministerial Resolution No.139/2011. Havana: Ministry of Education; 2012. 7 p.

30. Sex education and prevention of STIs and HIV/AIDS from the gender, sexual rights and sociocultural. Methodological guidelines. Secondary education, high school, vocational and adult Technique. Havana: Ministry of Education; 2018.

31. Rodriguez Carbajal C, G Ma Torres Cueto. Methodology for the development of the movement "School for Health". Havana: Editorial Molinos Trades SA; 2004. 225 p.

32. Cortes Alfaro A, García Roche R, Z Lazo González, et al. Intervention in knowledge, beliefs and risk perception about sexually transmitted infections and HIV/AIDS. Medicentro Electronics. 2014;18(1):28-35. 